\title{
Description and validation of a novel score (Flow Index) as a clinical indicator of the level of respiratory support to children on high flow nasal cannula.
}

\author{
sandeep tripathi ${ }^{1}$, Jeremy Mcgarvey ${ }^{2}$, Nadia Shaikh ${ }^{1}$, and Logan Meixsell ${ }^{2}$ \\ ${ }^{1}$ University of Illinois College of Medicine at Peoria \\ ${ }^{2} \mathrm{OSF}$ Saint Francis Medical Center
}

December 1, 2020

\begin{abstract}
Objective: Describe \& validate flow index $(\mathrm{FiO} 2 \times$ flow rate/weight $)$ to report the degree of respiratory support to children on high flow nasal cannula (HFNC) Methods: Retrospective chart review. Children managed with HFNC from 01/01/15 to $12 / 31 / 19$. Variables included in the flow index (weight, FiO2, flow rate) and outcomes (hospital and ICU length of stay [LOS], escalation to the ICU) extracted from medical records. Max flow index defined by the earliest timestamp when patients FiO $2 \times$ Flow rate was maximum. Step-wise regression used to determine the relationship between outcome (length of stay and escalation to ICU) and flow index Results: 1537 patients met the study criteria. Median 1st and maximum flow index of the population 24.1 and 38.1, respectively. Both 1st and maximum flow indexes showed a significant correlation with the LOS (r 0.25 and 0.31 ). Correlation for the index was stronger than that of the variables used to calculate them and remained significant after controlling for age, race, sex, and diagnoses. Mild, moderate, and severe categories of 1st and max flow index derived using quartiles and showed significant age and diagnosis independent association with LOS. Patients with 1 st flow index $>20$ and maximum flow index $>59.5$ had increased odds ratio of escalation to ICU (OR 2.39 and 8.08). The 1st flow index had a negative association with rapid response activation. Conclusions: Flow index is a valid measure for assessing the degree of respiratory support for children on HFNC. High flow index associated with longer hospital LOS and the risk of escalation to ICU.
\end{abstract}

\section{Introduction}

High Flow Nasal Cannula (HFNC) is increasingly utilized to provide non-invasive support to children with acute respiratory failure ${ }^{1,2}$. As the science behind HFNC matures and the evidence of its benefit accumulates ${ }^{3,4}$, it is also imperative to identify a scoring system to assess the level of respiratory support with HFNC. The score typically used to assess the degree of respiratory failure for intubated patients (oxygenation index $)^{5}$ would not apply for a patient on HFNC because of the absence of the essential variable of mean airway pressure. S/F Ratio ${ }^{6}$ has limited application for patients on HFNC as most patients are expected to have normal oxygen saturations (are escalated to a higher level of support if not able to maintain normal saturation). In adult literature, a scoring system of S.F. ratio/respiratory rate (ROX score) has been described to discriminate between patients who would succeed on HFNC (as described by the ability to avoid intubation $)^{7,8}$. However, the respiratory rate in children is a very unreliable indicator as it varies with age as well as with periods of agitation.

HFNC provides two degrees of support to the patient, (1) the flow rate, which presumably provides PEEP and assists in lung expansion ${ }^{9},(2)$ the $\mathrm{FiO} 2$, which provides support with oxygenation. For children, weight also becomes an essential factor in determining the level of support. Thus, to accurately represent the respiratory support level, all three variables must be taken into account. This complexity creates an opportunity to develop a single score that would \soutto represent respiratory support to the patient. 
We want to propose a new equation, which we would call a flow index. This equation includes the level of the patient's flow in liters/minute multiplied by the fraction of inspired oxygen (percentage)/weight (kilograms) or $(\mathrm{FiO} 2 \times$ Flow rate/Weight). We would like to describe this as a communication tool in describing the level of patient support and also for its ability to discriminate patients who may require escalation of care to the ICU, intubation, or a call for rapid response team (called as Pediatric Emergency Response Team [PERT] at our institution). This study's primary objective was to identify the association of the first and maximum flow index of a patient on HFNC with the severity of illness (using surrogate of hospital length of stay). The secondary objective was to identify the sensitivity and specificity of a threshold value of first and maximum flow index to predict escalation of care to ICU and PERT.

\section{Materials and Methods}

In this single-center retrospective chart review, we identified all children ( 0 to 17 years) who required HFNC therapy for respiratory support during their hospitalization at the Children's Hospital of Illinois/OSF Saint Francis Medical Centre in Peoria from 01/01/2015 to 12/31/2019. Children's Hospital of Illinois is a 136-bed tertiary referral hospital with a 16-bed Pediatric Intensive Care Unit (PICU). HFNC support is provided at all hospital floors. Hospital also has a robust PERT system, triggered by the Pediatric Early Warning (PEW) score threshold ${ }^{10}$ or nurse/parent concerns. All data were extracted by automated chart review from the electronic medical records. The study was reviewed and approved by the Institutional Review Board with a waiver of the informed consent.

\section{Study inclusion and exclusion criteria}

Patients with congenital malformations of the circulatory system, severe sepsis with septic shock, chronic respiratory failure, obstructive sleep apnea, cerebral palsy, pulmonary hypertension, and tracheostomy were identified based on their respective ICD 9/10 codes and excluded from the study. These patients were excluded as their expected titration of oxygen, and flow rate may be different from titration in children not with the aforementioned comorbidities. Patients who died during their hospitalization were excluded as the primary outcome variable was hospital length of stay. Patients who had any missing variable required for the calculation of the flow index (FiO2, flow rate, or the weight) and patients who had a gap of more than 24 hours between two chartings of the flow rate of HFNC were also excluded (as it was not possible to differentiate whether these patients had a gap of HFNC [stopped and then restarted], or if the charting was incomplete).

\section{Study variables}

The variables extracted from the EMR included age (in months), sex, race (classified as African American, White or others), body mass index, admitting diagnoses (sub-classified into organ system categories, and further as diagnostic categories of asthma, pneumonia, and bronchiolitis), and patient's initial admitting unit (first non-emergency room department). Age was categorized as Infant ( $<2$ years), Child ( 2 years to $<12$ years), and Adolescent (12 to 17 years). First and maximum recorded FiO2 (in percentage), oxygen flow rate of HFNC (lts/minute) were extracted from respiratory charting in the EMR flow sheet. The outcome variables included hospital length of stay, ICU length of stay (for patients admitted to the ICU), intubation (yes/no), escalation of care (transfer to the ICU after initial admission to the general pediatric/intermediate care floors), PERT activation and the duration of HFNC therapy. The exact timestamp of patients arrival to the hospital, escalation to the intensive care unit, PERT activation, intubation, as well as time stamp of the first Flow Index calculation as well as day/time during the patients HFNC therapy when the product of patients FiO2 and flow rate was maximum was also obtained. The unit for inclusion in the study was an admission. If the patient was admitted multiple times during the study, each admission was counted separately.

\section{Statistical analysis}

Standard descriptive and comparative analysis was performed with data presented as mean \pm standard deviation (S.D.) or median with interquartile range (IQR) for continuous variables and number with percentage for 
categorical variables. Simple regression was used to determine the relationship between the outcome (length of stay) and the individual components of the flow index; weight, FiO2, oxygen flow rate, the product of flow rate and $\mathrm{FiO} 2$, as well as the flow index itself, to determine the strength of the relationship. Flow index was also included in the step-wise multiple regression model to determine its significance after controlling for additional potentially confounding variables (age, gender, race, and diagnostic categories). Due to positively skewed data on length of stay, all analysis of length of stay was conducted after log transformation. The geometric mean length of stay is reported due to the use of log transformation. In addition to examining the relationship between numeric flow index and the length of stay, flow index was also grouped by quartiles to determine if a specific range of the score was related to the length of stay (Group one [Green]; first quartile, Group two [yellow]; second and third quartile, and Group three [Red], fourth quartile). Separate analysis was conducted for the first and maximum flow index. Strength of association of ICU length of stay with first and maximum flow index of patients who had initial admitting unit was ICU was also calculated.

For patients who required escalation to the ICU, after admission to the general pediatric floor and patients who required PERT activation, analysis of the relationship between first and maximum flow index and the event's binary occurrence (escalation to ICU or PERT ) was conducted by logistic regression. For this analysis, patients whose index was recorded (first or maximal) after the event (ICU transfer or PERT) were excluded. The R package "cutpointr" was used to determine the optimal flow index cut point, "threshold", for the classification of transfer or PERT by maximizing the sum of sensitivity and specificity. Because of the low numbers of adolescents in the high flow index category (only nine adolescents above the binary threshold of the high flow index for the first flow index and only one for the maximal flow index), adolescents were excluded from the analysis of the prediction of ICU transfer/PERT based on flow index. LASSO and step-wise regression were used to select variables to include the flow index threshold in multivariate logistic regression on ICU transfer and PERT. Potential predictors included age group, admission diagnostic group, sex, and race. All statistical analysis was performed using the open-source statistical program $\mathrm{R}$ version 4.0.0) and JMP Pro (version 14.2), SAS Inc (Cary, NC), against a two-sided alternative hypothesis with a significance level of $5 \%$.

\section{Results}

A total of 2209 patients met the inclusion criteria during the study period (01/01/2015 to 12/31/2019). Out of these patients, 672 were further excluded. A total of 1537 patients were included in the analysis. Out of these patients, $352(22.9 \%)$ were directly admitted to the ICU from the emergency department, and 270 (18.5\%) patients required escalation to the ICU. 162 patients (10.5\%) required intubation and $288(18.7 \%)$ patients had a PERT activation. (Figure 1)

\section{Study demographics}

The sample was $57 \%(873 / 1537)$ male and $64 \%$ (990/1527) Caucasian with a median age of 20 (IQR 7, 60) months and a median weight of 11.2 (IQR 7.3, 18.8) kg. The median hospital and ICU length of stay was 4.5 (IQR 2.9, 7.3) days and 2.2 (IQR 1.0, 5.1) days respectively. The median duration of HFNC therapy was 45 (IQR 23, 75) hours. The most common admitting diagnosis system was respiratory $(80.6 \%)$. Among patients in the respiratory category, $230(14.9 \%)$ had a diagnosis of asthma, 291 (18.9\%) had bronchiolitis, and $104(6.7 \%)$ had pneumonia. The whole study population's median first flow index was 24.1 (IQR 13.5, 42.9), while the median maximal flow index was 38.1 (IQR 20.6, 64.8). (Table 1)

\section{Relationship between flow index and hospital/ICU length of stay}

Table No 2 shows the results of the simple linear regression between log-transformed hospital and ICU length of stay and the first and maximum recorded weight $(\mathrm{kg}), \mathrm{FiO} 2$ ( percentage), oxygen flow rate (liters/min), FiO2 multiplied by oxygen flow rate and the $(\mathrm{FiO} 2 *$ Flow rate/weight). For hospital length of stay, weight was not significantly related to the length of stay. All other variables showed a significant positive relationship with the length of stay, indicating that an increase in these variables would be associated with an increased length of stay. Both the first and max flow index had a stronger correlation coefficient than the variables used to calculate them. The first flow index had a correlation coefficient of 0.25 , and the maximum flow 
index having a correlation coefficient of 0.31 .

Among patients who had initial admission to the ICU, patients' weight had a significant negative association with the ICU length of stay. Except for the first recorded FiO2, all other variables were significantly associated with the ICU length of stay. As with the hospital length of stay, both first and maximum flow index had a stronger correlation ( 0.21 and 0.29 respectively) with the ICU length of stay than the index's constituent variables. (Table 2)

The first and maximum flow indexes were included in the multivariate regression along with potential confounders of age, race, sex, and diagnostic categories (asthma, pneumonia, and bronchiolitis). LASSO and step-wise linear regression were used to eliminate variables that did not contribute to the model's performance. Both first and maximum flow index remained significantly associated with the increased hospital length of stay after controlling for other variables. The first flow index relationship with hospital length of stay was independent of age and diagnostic categories (impact of first flow index on the length of stay was similar for each age group and diagnostic category). However, there was a significant positive $(\mathrm{p}=0.037)$ interaction between maximum flow index and child age group. Infants ( $<2$ years) had higher LOS than child age group ( 2 to 12 years), but the difference between LOS for infants and child decreased as flow Index increased, until it reversed at very high max flow Index. (SDC Table)

\section{Mean length of stay of different flow index categories}

The first and maximum flow index were categorized into three subgroups based on quartiles. The cutoff point for the first flow index was Green $<13$; Yellow 13 to $<43$; Red $>43$, and the cutoff for the maximum flow index was Green $<20$, Yellow 20 to $<64$, and Red $>=64$. Results of simple linear regression on logtransformed length of stay and first and maximum flow index categories are shown in Table No 3. There was a statistically significant difference in the geometric mean length of stay between green and yellow/red categories for both first and maximum flow index.

On multiple regression using the same control variables as described previously (age, race, sex, and diagnostic categories [asthma, pneumonia, and bronchiolitis] and using flow index category, rather than continuous flow index variable), the significant difference of green versus yellow/red categories persisted for both first and maximum flow index. No significant interactions were found between the first or maximum flow index category and other included predictors indicating that the impact of first and maximum flow index categories on length of stay was independent of age and admitting diagnosis category of asthma/pneumonia or bronchiolitis.

\section{Prediction of transfer to ICU (Children 12 years and under)}

A first flow index value $>20$ was significantly more likely to be associated with an ICU transfer (odds ratio 2.4, $95 \%$ C.I 1.7, 3.5) with AUC of 0.60 , a sensitivity of $71.7 \%$, and a specificity of $48.8 \%$. Patients with a first flow index $>20$ were expected to have an ICU transfer rate of approximately $23.4 \%$ compared to $11.3 \%$ for those with the first flow index $<20$. Similarly, a maximum flow index greater than 59.5 was significantly associated with an increased likelihood of being transferred to the ICU (odds ratio 7.6, 95\% C.I 4.8, 12.5,) with an AUC of 0.73 , the sensitivity of $65.9 \%$ and specificity of $79.8 \%$. Patients with the maximum flow index $>59.5$ had an ICU transfer rate of $25.9 \%$ compared to $4.4 \%$ for patients with the maximum flow index $<59.5$.

On multivariable LASSO regression, using additional variables of age group, race, sex, and diagnostic categories of asthma, bronchiolitis, or pneumonia, no other variables were found to contribute to the model's performance above having first flow index $>20$ or maximum flow index $>59.5$. In a logistic regression with a first flow index over 20, age group, race, sex, asthma, bronchiolitis, and pneumonia as predictors, a first flow index over 20 was the only variable found to be significantly associated with an increased likelihood of being transferred to the ICU (odds ratio 2.34, 95\% C.I 1.62, 3.42, p <0.001). Similar results were found when the maximum flow index over 59.5 was included with age group, race, sex, asthma, bronchiolitis, and pneumonia. A flow index over 59.5 was the only variable significantly related to an increased likelihood of a transfer to the ICU (odds ratio 8.08, $95 \%$ C.I 4.9, 13.7, p <0.001). (Table No 4 and Figure 2) 


\section{Flow Index and PERT}

All the variables associated with the first flow index, except weight, were significantly associated with a decrease in PERT's likelihood; however, the first flow index resulted in the highest area under the curve (AUC) at 0.58. None of the components used to calculate the maximum flow index were significantly associated with PERT. The maximum flow index itself was also not significantly associated with PERT and produced an area under the curve of 0.50 . The maximum flow index was excluded from further analysis since it did not show a significant relationship to PERT.

The first flow index had a negative association with the incidence of PERT. A first flow index below 31 was significantly more likely to be associated with PERT (OR 2.54, 95\% C.I 1.77, 3.71, p<0.001) and produced an AUC of 0.59 with a sensitivity of $80.0 \%$ and a specificity of $38.8 \%$. Inclusion of the race, sex, and age group variables in LASSO regression increased the AUC to 0.64.

\section{Discussion}

In this paper, we have described a novel single metric (flow index) to characterize the degree of respiratory support by HFNC in children. We have shown that the flow index is a valid measure to assess disease severity (using hospital length of stay as a surrogate marker). We have also categorized the first and maximum flow index values into mild, moderate, and severe categories ( described as green, yellow, and red) and further identified the sensitivity and specificity of predicting escalation to ICU and PERT using flow index cutoffs.

Most prior studies on HFNC have focused on its ability to provide non-invasive respiratory support to children and adults ${ }^{1}$. HFNC has also been shown to decrease hospital length of stay and requirement of intubation ${ }^{11}$. To the best of our knowledge, no study has investigated methods to quantify respiratory support provided by HFNC. The idea behind the flow index is relatively simple. It is also easy to calculate at the bedside and trend over time. It attempts to answer the question of whether a $10 \mathrm{~kg}$ patient who in the morning was on $10 \mathrm{~L} 30 \%$ and now on $6 \mathrm{~L} 50 \%$ is getting the same degree of respiratory support or less/more.

The red, yellow, green categories of flow index provide meaning to the flow index values. They are similar to the oxygenation index ${ }^{12}$ and $\mathrm{PaO} 2 / \mathrm{FiO}^{13}{ }^{13}$ categorization of acute respiratory distress syndrome (ARDS). Flow index, as described, only provides information on the support patient is receiving and does not include any patient response variables like saturations or $\mathrm{PaO}$. This contrasts the flow index from the indexes mentioned earlier. The majority of patients on HFNC have normal saturations (as they are escalated to a higher level of support otherwise), thus adding saturation to the score does not add much value. While $\mathrm{PaO} 2$ may vary between different patients, most patients on HFNC do not have arterial lines, and incorporating $\mathrm{PaO} 2$ in the formula would limit its applicability.

We have used hospital and ICU length of stay as surrogate markers for the severity of illness. However, length of stay is a crude index for severity as it can be impacted by factors unrelated to the illness ${ }^{14}$. Patients with mortality were excluded from our analysis. Mortality in pediatric patients is very $\operatorname{low}^{15}$, and assessment of the impact on mortality would require an extremely large dataset. Intubation can serve as a definitive endpoint for the failure of HFNC therapy. Unfortunately, intubation prediction could not be calculated in our study as precise intubation times were not reliably charted in our EMR.

The impact of age in the performance of the flow index deserves special mention. The score uses the denominator of weight. While the generally accepted practice of HFNC is to use 2 liters per minute per kilo ${ }^{2}$, this is not always possible for older children. For example, it would be relatively common to use a 6 liters $/ \mathrm{min}$ flow rate for a $3 \mathrm{~kg}$ infant; it would be uncommon to use 100 liters $/ \mathrm{min}$ for a $50 \mathrm{~kg}$ adolescent. Thus, the flow index would be lower for older patients. The flow index category (of red/yellow/green), however, had a significant difference with the length of stay even after controlling for age categories, so it would be applicable for all age groups.

The flow index value of 31 for the first flow index and 59.5 for the maximum flow index provides a cutoff for maximum AUC for the risk of ICU admission. Even though the odds ratio for escalation to ICU using the cutoffs are high (2.34 and 8.08), sensitivity and specificity on linear scales are low. However, they 
are applicable for all age groups (12 and under) and diagnostic categories of bronchiolitis, asthma, and pneumonia. Thus, they can potentially be utilized as decision points for escalating care on patients on HFNC on general pediatric floors.

The negative association of the first flow index with the PERT call was an unexpected observation. Although PERT calls are based on various patient/provider factors ${ }^{16,17}$, one of the criteria is an escalation of patients' oxygenation or flow rate. Thus, if the patient were started on a very low degree of respiratory support (lowflow index) and required escalation, later on, the chances of him having a PERT call would be higher. This association, however, should be evaluated further in future studies.

Flow index, as described in our study, has several limitations. Even though the flow index performs better than the individual component, the overall correlation of the flow index with the length of stay is small. The flow index's discriminating ability diminishes with age, although the flow index categories maintain an independent association with length of stay irrespective of the age categories. Secondly, the score gives equal weight to the flow rate in liters per minute and the fraction of inspired oxygen. It thus does not discriminate between hypoxemic and hypercapnic respiratory failure. For example, a patient with asthma might need a higher flow rate but low $\mathrm{FiO} 2$ compared to a patient with ARDS who may need both a higher flow rate and FiO2. Thus, the score for the patient with asthma and ARDS may not be comparable to assess the severity of illness. Our study, however, showed that the association of flow index with the length of stay and risk of ICU admission persisted even after controlling for the three main diagnostic categories. Escalation to ICU and PERT are very hospital policy sensitive parameters and may not have external validity.

\section{Conclusion}

Flow index (FiO2 $\times$ Flow rate/Weight) can be used as a metric for rapid assessment and communication of the degree of respiratory support in children on high flow nasal cannula. Absolute and mild/moderate/severe categories have a significant association with the hospital length of stay. Further studies should look at independent validation in a different patient population.

\section{References}

1. Coletti KD, Bagdure DN, Walker LK, Remy KE, Custer JW. High-flow nasal cannula utilization in pediatric critical care. Respiratory care. 2017;62(8):1023-1029.

2. Ricard J. High flow nasal oxygen in acute respiratory failure.Minerva Anestesiol. 2012;78(7):836-841.

3. Rochwerg B, Granton D, Wang D, et al. High flow nasal cannula compared with conventional oxygen therapy for acute hypoxemic respiratory failure: a systematic review and meta-analysis. Intensive care medicine. $2019 ; 45(5): 563-572$.

4. Lin J, Zhang Y, Xiong L, Liu S, Gong C, Dai J. High-flow nasal cannula therapy for children with bronchiolitis: a systematic review and meta-analysis. Archives of disease in childhood.2019;104(6):564-576.

5. Trachsel D, McCrindle BW, Nakagawa S, Bohn D. Oxygenation index predicts outcome in children with acute hypoxemic respiratory failure.American journal of respiratory and critical care medicine.2005;172(2):206-211.

6. Mayordomo-Colunga J, Pons M, López Y, et al. Predicting non-invasive ventilation failure in children from the $\mathrm{SpO} 2 / \mathrm{FiO} 2$ (SF) ratio.Intensive care medicine. 2013;39(6):1095-1103.

7. Roca O, Caralt B, Messika J, et al. An index combining respiratory rate and oxygenation to predict outcome of nasal high-flow therapy. American journal of respiratory and critical care medicine.2019;199(11):13681376.

8. Roca O, Messika J, Caralt B, et al. Predicting success of high-flow nasal cannula in pneumonia patients with hypoxemic respiratory failure: The utility of the ROX index. Journal of critical care.2016;35:200-205. 
9. Parke RL, McGuinness SP. Pressures delivered by nasal high flow oxygen during all phases of the respiratory cycle. Respiratory care. 2013;58(10):1621-1624.

10. Duncan H, Hutchison J, Parshuram CS. The Pediatric Early Warning System score: a severity of illness score to predict urgent medical need in hospitalized children. Journal of critical care.2006;21(3):271-278.

11. Wing R, James C, Maranda LS, Armsby CC. Use of high-flow nasal cannula support in the emergency department reduces the need for intubation in pediatric acute respiratory insufficiency. Pediatric emergency care. 2012;28(11):1117-1123.

12. Cheifetz IM. Pediatric ARDS. Respiratory care.2017;62(6):718-731.

13. Ferguson ND, Fan E, Camporota L, et al. The Berlin definition of ARDS: an expanded rationale, justification, and supplementary material.Intensive care medicine. 2012;38(10):1573-1582.

14. Brasel KJ, Lim HJ, Nirula R, Weigelt JA. Length of stay: an appropriate quality measure? Archives of Surgery.2007;142(5):461-466.

15. Pollack MM, Holubkov R, Funai T, et al. Pediatric intensive care outcomes: development of new morbidities during pediatric critical care.Pediatric critical care medicine: a journal of the Society of Critical Care Medicine and the World Federation of Pediatric Intensive and Critical Care Societies. 2014;15(9):821.

16. Van Voorhis KT, Willis TS. Implementing a pediatric rapid response system to improve quality and patient safety. Pediatric clinics of North America. 2009;56(4):919-933.

17. Chen J, Bellomo R, Hillman K, et al. Triggers for emergency team activation: a multicenter assessment. Journal of critical care.2010;25(2):359. e351-359. e357.

\section{Hosted file}

Table 1.pdf available at https://authorea.com/users/380450/articles/496434-descriptionand-validation-of-a-novel-score-flow-index-as-a-clinical-indicator-of-the-level-ofrespiratory-support-to-children-on-high-flow-nasal-cannula

\section{Hosted file}

Table 2.pdf available at https://authorea.com/users/380450/articles/496434-descriptionand-validation-of-a-novel-score-flow-index-as-a-clinical-indicator-of-the-level-ofrespiratory-support-to-children-on-high-flow-nasal-cannula

\section{Hosted file}

Table 3.pdf available at https://authorea.com/users/380450/articles/496434-descriptionand-validation-of-a-novel-score-flow-index-as-a-clinical-indicator-of-the-level-ofrespiratory-support-to-children-on-high-flow-nasal-cannula

\section{Hosted file}

Table 4.pdf available at https://authorea.com/users/380450/articles/496434-descriptionand-validation-of-a-novel-score-flow-index-as-a-clinical-indicator-of-the-level-ofrespiratory-support-to-children-on-high-flow-nasal-cannula 

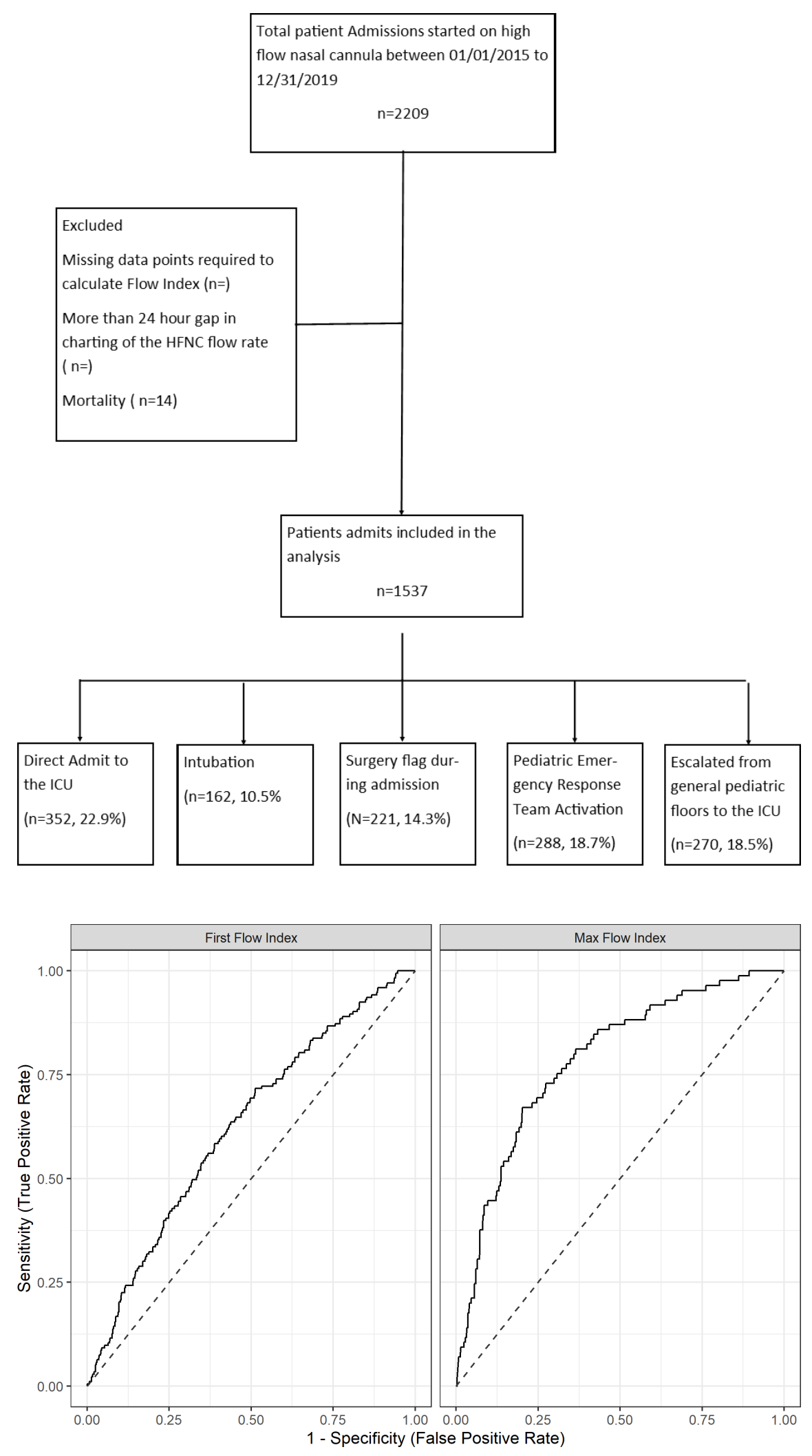